\title{
Erratum to: Effect of Conductivity Type and Doping Level of Silicon Crystals on the Size of Formed Pore Channels during Anodic Etching in Hydrofluoric Acid Solutions
}

\author{
G. G. Zegryaa, ${ }^{a, *}$ V. P. Ulin ${ }^{a}$, A. G. Zegryaa ${ }^{a}$ N. V. Ulin ${ }^{a}$, \\ V. M. Frayman ${ }^{a}$, and Yu. M. Mikhailov ${ }^{b}$ \\ ${ }^{a}$ Ioffe Institute, St. Petersburg, 194021 Russia \\ ${ }^{b}$ Institute of Problems of Chemical Physics, Russian Academy of Sciences, Chernogolovka, Moscow oblast, 142432 Russia \\ *e-mail: zegrya@theory.ioffe.ru \\ Received November 16, 2020
}

DOI: $10.1134 / \mathrm{S} 106378422102016 \mathrm{X}$

The list of authors should read as follows:

G. G. Zegrya ${ }^{a}$, , V. P. Ulin ${ }^{a}$, A. G. Zegrya ${ }^{a}$, N. V. Ulin ${ }^{a}$, V. M. Frayman ${ }^{a}$, and Yu. M. Mikhailov ${ }^{b}$

The original article can be found online at https://doi.org/10.1134/S1063784219100268 